\title{
Based on PCI Software Day Draw Satellite is Projective Like Making Technology Discussed in This Paper
}

\author{
Ming Zhao ${ }^{1}$, Rui Wang ${ }^{1, a}$, Yongming $\mathrm{Xu}^{2}$ and Xiaochun $\mathrm{Xu}^{2}$ \\ ${ }^{1} 32023$ troops, Dalian, Liaoning, China \\ ${ }^{2}$ Guo Fang Da Xue, Shanghai, Shanghai China
}

\begin{abstract}
This article introduces the use of PCI software production day draw satellite is projective like production process, analyzes the key technologies in the production, summarizes the Suggestions to improve the quality of results, strengthen quality control method is proposed, for the day draw satellite orthogonal projection as mass production work has carried on the beneficial exploration.
\end{abstract}

\section{The introduction}

Digital orthophoto image (DOM) is an important 4D surveying and mapping product with high precision, rich information, intuitive and lifelike features ${ }^{[1]}$, which has been widely used in various industries. There are many methods of making digital orthophoto images, most of which require accurate field control point data, high production cost and long production cycle. Day painted a satellite is the first shows the three-dimensional mapping satellite, $2 \mathrm{~m}$ high-resolution panchromatic images can be acquired at the same time, $10 \mathrm{~m}$ resolution of multispectral images and $5 \mathrm{~m}$ resolution three linear array, and adopting control point measurement technology, in the process of making orthographic like, does not need to be field measurement control points, own RPC parameters can be directly read image, is to make orthogonal projection as the preferred data source.

In the production of large-scale digital orthophoto images, there are many problems, such as complex process, poor production quality and low production efficiency, which affect the modularization, scale and standardization of digital orthophoto images. PCI Geomatica software is capable of mass image processing in the production process of orthophoto satellite, which has high absolute precision and edge precision. Compared with other similar orthophoto image processing software, it has obvious advantages in processing over thousands of orthophoto images. This paper, combined with PCI Geomatica software, summarizes the process of producing digital orthophoto images based on satellite images. This method can greatly improve the production efficiency and data quality of orthophoto images, and has been successfully applied to the construction of basic geographic information database in China.

\footnotetext{
a Corresponding author: author@e-mail.org
}

\section{PCI Geomatica software}

PCI Geomatica software has a strong development background and is directly supported by the Canadian government and the Canadian remote sensing center. It is a banner product in the field of geospatial information ${ }^{[2]}$, mainly applied in the fields of remote sensing image processing, professional radar data analysis, GIS/ spatial analysis, mapping and digital photogrammetry. As a pioneer of image processing software system, PCI Geomatica software is the remote-sensing image processing software with the best orthophoto processing effect and the highest precision in current image processing software. With a unique image fusion method, it is the best fusion method recognized in the world at present. It can retain the color information and space information of the image to the maximum extent, and the image after fusion is closer to reality. It provides the functions of automatic inlay, automatic uniform and automatic registration, which greatly improves the production efficiency and production quality of orthophoto images.

\section{Rational function model}

\subsection{Features of rational function model}

The correction model of satellite image mainly includes polynomial model, rational function model and strict physical model. Due to the secrecy requirement of the satellite, its orbit and technical parameters are not open to the public, so the corresponding physical sensor model cannot be established, while the rational function model is applicable to different sensors and supports any coordinate system, which can provide sufficient 
computing speed for real-time mapping operation. When dealing with the satellite images, the rational function model provided by PCI Geomatica software is usually selected to replace the strict imaging model.

\subsection{Definition of rational function model}

Rational function model is a generalized imaging model which is independent of specific sensor and describes the relationship between image points and corresponding ground points in simple form. The rational function model is defined as follows:

$$
\begin{aligned}
& \mathrm{S}=\mathrm{Ss}\{\mathrm{P} 1(\mathrm{Xn}, \mathrm{Yn}, \mathrm{Zn}) / \mathrm{P} 2(\mathrm{Xn}, \mathrm{Yn}, \mathrm{Zn})\}+\mathrm{S} 0 \\
& \mathrm{~L}=\mathrm{Ls}\{\mathrm{P} 3(\mathrm{Xn}, \mathrm{Yn}, \mathrm{Zn}) / \mathrm{P} 4(\mathrm{Xn}, \mathrm{Yn}, \mathrm{Zn})\}+\mathrm{L} 0
\end{aligned}
$$

$(\mathrm{Xn}, \mathrm{Yn}, \mathrm{Zn})$ is like point coordinates, $(\mathrm{S}, \mathrm{L})$ is the image point coordinate after dealing with the translation and narrowing the amplification of coordinates, (S0, L0) is a standardized parameters P1 (Xn, Yn, Zn), P2 (Xn, Yn, $\mathrm{Zn}$ ), P3 (Xn, Yn, Zn), P4 (Xn, Yn, Zn) is (Xn, Yn, Zn) polynomial expression, the formula is described in the object space space coordinate to like the space coordinate transformation, the ground point $3 \mathrm{~d}$ coordinate $(\mathrm{Zn})$ available DEM elevation values in the data, And rational function system and standardized parameters are stored in a *. The RPC file, the *. The RPC file, when buying day draw satellite image data will be provided with images, the user in the known ground point $3 \mathrm{~d}$ coordinate and standardize parameter, calculated can image point coordinate $(\mathrm{S}, \mathrm{L})$, which is based on PCI Geomatica software production day draw satellite is projective like correct principle.

\section{The process of making an orthophoto of an astronomical satellite}

In this paper, by using PCI Geomatica software, a study area in 2013 days in domestic satellite $2 \mathrm{~m}$ highresolution panchromatic image, $10 \mathrm{~m}$ resolution of multispectral images as the data source, rational function model is chosen as the image of the correct model, the software will automatically extract the $*$. In the original file RPC file, and use the existing DEM data to correct panchromatic images and multi-spectral image registration and fusion, finally put all the Mosaic image, after inspection qualified can obtain high quality day draw orthogonal projection image.

\subsection{Preprocessing of original images}

Due to the interference of atmospheric refractive error, thin cloud and haze, etc., the original sky map satellite image will suffer a certain loss, which requires the preprocessing of the original sky map satellite image before the normal projection image is made. The brightness and contrast can be adjusted by using Photoshop to make the image have moderate contrast, clear texture and closer color to real ground objects.

\subsection{Make DEM}

DEM data is the key to determine the quality of orthophoto images. The higher DEM accuracy, the less stretching and deformation of orthophoto images will occur. Although the Internet now provides free 1:50,000 scale DEM data worldwide, the accuracy is poor, and some areas are highly deformed and present potential is not high. It is suggested to use contour data within the study area to automatically generate DEM data in VirtuoZo software with high precision and small deformation, which is conducive to the production of high-quality orthophoto data.

\subsection{Correction of panchromatic images}

By using PCI Geomatica software, the rational function model is set up, DEM data is introduced, *.rpc files provided with satellite images are imported, and the panchromatic image of the satellite with $2 \mathrm{~m}$ resolution is corrected.

\subsection{Image Fusion}

The pre-processed multispectral image is matched with the corrected panchromatic image. The image after fusion has rich color information, and more spectral information is added at the same time. The image color is closer to real ground objects and the texture is clearer.

\subsection{Image Mosaic}

Image Mosaic is a seamless Mosaic of multiple orthophoto images by position coordinate information. Although PCI Geomatica software provides automatic image Mosaic function, it also requires manual inspection of the Mosaic line, and modification of the splicing line manually if there is any problem. For images taken at different times, the edge of the image is very complicated, which needs to be executed in strict accordance with the standards of technical design to ensure the quality of orthophoto images.

\subsection{Quality inspection}

The quality inspection of orthophoto image mainly includes precision inspection, clarity inspection and integrity inspection. Precision check is to use the control point or ground object vector data to superpose with the orthophoto image map and select the feature point to check the position accuracy. The definition check is mainly to check whether the image is clear, whether the tone is uniform, whether the texture features are obvious, and whether the color difference is moderate. Integrity inspection mainly examines the stretching and distortion of ground objects, as well as the edge dislocation and deformation caused by Mosaic lines.

\section{Suggestions for improving the quality of orthophoto images}




\subsection{Methods to solve the cloud cover area.}

When the satellite is shooting image, it will often encounter the situation of cloud interference. PCI Geomatica software has an atmospheric analysis module, which can set the corresponding distinguishing parameters according to the difference of spectral features and infrared features of cloud fog, and realize rapid extraction of cloud fog area. The processing of thin cloud and thin fog can be better handled by improving the spectral information of image itself, such as enhancing contrast and wavelet decomposition. At present, the processing of thick clouds and thick fog cannot be solved by changing the image itself. Only other image data can be used to repair, so as to guarantee the quality of orthophoto images.

\subsection{Improve DEM data accuracy}

DEM data automatically generated by contour lines have high accuracy, but some areas need to be modified and edited manually to reduce stretch, deformation and distortion in orthophoto images. For example, where features such as ridges and gullies are obvious, the description of automatically generated DEM data is not accurate enough. Only when feature lines are collected manually, can the ridge not be deformed and the shape of gullies be correct when image is corrected.

\subsection{Treatment of deformed ground objects}

In the process of image correction, ground objects in areas with large drop are prone to distortion and deformation. For example, Bridges, steep cliffs, and tall buildings all have different degrees of distortion and deformation. Normally, Photoshop can be used to cut the distorted ground objects on the original image, and then paste them onto the orthophoto image, and correctly process the edge area to ensure the color transition is natural and accurate.

\subsection{Editing of Mosaic lines.}

PCI Geomatica software has the function of automatic generation of Mosaic lines. Compared with the traditional method of manual collection of Mosaic lines, it can save a lot of work. However, in some places, manual intervention is needed to avoid the situation of ground object dislocation and color transition unnaturalness caused by wrong location of Mosaic lines collection. The editing line should follow the edge of the object as much as possible to avoid crossing the ground object. If it is impossible to avoid crossing the ground object, you should try to choose the position where the color transition is natural and the ground object is less deformed, so as to ensure that the Mosaic line has a uniform direction without crossing or discounting.

\section{Conclusion}

Based on PCI Geomatica software, the method of making orthophoto images of celestial mapping satellites has the advantages of simple operation, fast efficiency, high quality, high degree of product standardization and modularization, and can meet the production task of mass orthophoto data of satellites. Although a certain amount of manual intervention is still needed in production, we believe that with the continuous upgrade and update of PCI Geomatica software, the satellite sensor of tianmu is developing continuously. As well as the gradual maturity of non-ground control point measurement technology, it will be more conducive to the production of high quality satellite orthophoto data.

\section{References}

1. J Liu. Journal of Henan university of science and technology. J. 196-200, (2010)

2. S Chou. Jiangxi surveying and mapping. J. 35$37,(2007)$ 